\title{
Author Index Volume 38
}

(c) Australasian College of Physical Scientists and Engineers in Medicine 2015

\section{A}

Abbas, Sarmad 643

Abdel-Rahman, Wamied 425

Abdullah, Baharudin 721

Ackerly, Trevor 447

Ahmad, Rana Fayyaz 139

Ahmadi, Mohsen 603

Ahmadvand, Ali 241

Al-Enezi, Mamdouh S. 425

Al-Karmi, Anan M. 425

Allen, Barry J. 527

Alves, Andrew D. C. 151

Amin, Hafeez Ullah 139

Arsalan Naqvi, Syed Ali 721

Arumugam, S. 255

Asena, A. 289

Atyabi, Seyyed Abbas 581

Ayaz, Ali Asghar H. 425

Azhari, H. A. 381, 493

\section{B}

Badruddin, Nasreen 139, 721

Baggarley, S. 493

Bai, Yinwei 695

Baig, Mirza Mansoor 23

Bailey, Michael 205, 479

Baldwin, Zoë 205, 479

Baluti, Florentina 101

Barnes, Michael 39

Bashir, Saba 305

Beeksma, B. 255

Begg, Jarrad 561

Berk, Michael 157

Bibbo, Giovanni 71
Bien, Mauo-Ying 657

Bridge, Pete 369

Burrage, John 1

Butler, Duncan 151, 325

Butson, Martin 619

C

Caon, Martin 203, 689

Chalkia, M. T. 7

Charles, Paul H. 93, 289, 357

Chatziioannou, S. N. 7

Chaurasia, P. P. 381

Chen, Feng 777

Chen, Mingyang 627

Chen, Tom 619

Chen, Yang 345

Cheng, Yih-Lin 657

Cheung, K. Y. 381, 493

Chhom, S. 381

Chooi, Weng-Tink 139

Chu, Winnie C. W. 375

Cole, Andrew 151

Conheady, Clement F. 447

Connolly, Martin J. 23

Cranmer-Sargison, G. 357

Crowe, S. B. 357

Cutajar, D. L. 455

D

Dai, Peishan 271

Daliri, Mohammad Reza 241

Davenport, David 465

Deevband, Mohammad Reza 603

Deloar, Hossain M. 101 
Dempsey, Claire 689

Deng, Xuefei 217, 777

Deng, Ying-Yuan 709

DilipKumara, A. H. 493

Dowling, Jason 39, 561

Dunn, Leon 151

Dwaikat, Nidal 425

E

Ebrahimzadeh, S. A. 611

Efstathopoulos, E. P. 7

Elazab, Ahmed 627

Eldeib, Ayman 223

Endo, M. 381

F

Faeghi, F. 611

Fan, Min 271

Fang, Qiong 777

Fardid, Reza 83

Farrukh, S. 381, 493

Fogarty, Gerald 619

Fox, C. 283

Franich, Rick D. 55

Fukuda, S. 217

G

Gagliardi, Frank M. 447

Geethanjali, P. 331

Ghaffari, Ali 581

Ghahraman Asl, Ruhollah 465

Ghassemi, A. 63

GholamHosseini, Hamid 23

Gong, Xue-Hao 709

Gray, Alison 619

Greer, Peter B. 39, 561

Gregory, Kent J. 71

Gu, Junyi 755

Gu, Xiang 767

Guatelli, S. 455

\section{H}

Han, Y. 217, 381, 493

Haque, Mamoon 665

Hardcastle, N. 283

Harirchian, M. H. 611

Harris, Benjamin D. 399

Harris-Phillips, W. 129

Harty, Peter 325

Haryanto, F. 381

Hassani, Kamran 569

Hazabbah, Wan 721

Hellyer, James 619
Hill, B. 289, 503

Hill, Robin 619, 665

Holloway, L. 255, 543, 561

Hosain, Md. Kamal 157

Hsieh, Ming-Fa 109

Hsu, Ding-Yang 657

$\mathrm{Hu}$, Qingmao 627

$\mathrm{Hu}$, Shiyu 785

$\mathrm{Hu}$, Yining 345, 381

Hua, Wei 767

Huang, Huajun 109, 743

Huang, Wenhua 109, 743

Huang, Xian 709

Huang, Yanping 777

Huang, Yu-Xiang 767

Hui, Steve C. N. 377

Hussain, Muhammad 139

I

Inamura, K. 493

Izard, Michael 619

J

Jafari, S. 381

Jafari Moghadam Fard, Pooria 47

Jafri, S. M. A. 381

Jamshidi, N. 63

Jatoi, Munsif Ali 721

Jia, Fucang 627

Jiang, Anhong 777

Johnston, Peter 151

K

Kairn, Tanya 93, 289, 357, 503

Kamel, Nidal 139

Kardan, Mohammad Reza 603

Karimi, Alireza 569

Karimi, M. T. 63

Karimi Alavijeh, S. 611

Kastanis, Lazaros 369

Kaya, Yilmaz 435

Kaynak, Akif 157

Kenny, John 151

Khaleghi, Ali 551

Khalid, Shehzad 643

Khan, Farhan Hassan 305

Khor, R. 283

Kouzani, Abbas Z. 157

Krisanachinda, A. 217, 381, 493

Kron, Tomas 151, 381, 493

Kumar, Shivani 561

Kuo, Men 493

Kuppusamy, T. 381 
L

Lambert, Jonathan 561

Lancaster, Craig M. 55, 119

Langton, C. M. 289, 357

Lansley, Stuart P. 101

Lawson, J. M. 129

Lee, C. C. 493

Lee, Hsin-Chien 657

Lee, James 619

Lehmann, Joerg 151

Li, Guanglin 627

Li, Jun 676

Li, Weiping 785

Li, Xiaodong 627

Li, Xinchun 271

Li, Zheng 695

Lim, A. 283

Lim, Karen 561

Lin, Huixin 695

Liu, Jin 709

Liu, Liping 785

Liu, Li-Qin 767

Liu, Wei-zong 709

Lu, Jun 709

Lubis, L. E. 381

Luo, Limin 345

Luvsan-Ish, A. 381

Lye, Jessica 151, 325

\section{M}

Madadi, A. 611

Mahmoud, Shahenda 223

Malik, Aamir Saeed 139, 721

Marcu, Loredana G. 531

Marsh, S. 493

Mason, Natasha 55

Masood, K. 473

Mauldon, G. F. 381

Maung, S. O. 381

McLean, I. D. 543

Mehrpouyan, Mohammad 83

Meng, Jinyu 345

Menk, Fred 39

Meyer, Juergen 101

Middlebrook, N. D. 289, 503

Millar, Jeremy L. 55

Mishra, Alok 413

Moeini, Mahdi 551

Mohammadi, Mohammad Reza 551

Mollakazemi, Mohammad Javad 581

Momennezhad, Mehdi 465

Moradi, Mohammad Hassan 47

Morales, Johnny 665
Mossayebi, A. R. 63

Moutrie, Vaughan 93

Moylan, R. 289

N

Nadarajan, R. 731

Nademi, Hossein 83

Naemi, R. 63

Najafi, Mohsen 603

Nasrabadi, Ali Moti 551

Nasseri, Shahrokh 465

Nelligan, Raelene 205, 479

Nelson, V. K. 543, 619

Ng, K. H. 217, 381, 493

Nguyen, T. C. 381

Nguyen, X. C. 493

Nikiforidis, G. C. 7

Niknazar, Mohammad 581

Nilsson, Sanna 399

Nourbehesht, L. K. 455

O

Oliver, Chris 325

Ostwald, Trish 479

Ouyang, Hanbin 109, 743

P

Pack, Darren 369

Panettieri, Vanessa 55

Parach, Ali Asghar 465

Parker, Jacqui C. 369

Pattison, John E. 71

Pawiro, S. A. 381

Peralta, A. 381

Pirbhulal, Sandeep 709

Poole, C. M. 119, 399

Q

Qamar, Usman 305

R

Rahimian, N. 611

Rajapakse, Satya 619

Ramanathan, Ganesan 325

Rattanavoang, Somkhit 619

Ravindran, P. 493

Rawal, Kirti 509

Ren, Lijie 785

Rezaei, Farshid 569

Rijken, J. D. 129

Rodriguez, L. V. 217, 493

Rosenfeld, Anatoly B. 93, 455

Round, W. H. 7, 217, 377 


\section{S}

Saber, Sassan 47

Saeed, M. K. 299

Saini, B. S. 509, 671

Saini, Indu 509

Saligheh Rad, H. 611

Samy, Sherif 223

Sarasanandarajah, S. 381

Serdar Bascil, M. 229

Sethi, Gaurav 671

Sharif, F. 593

Sharififar, Mohammad 241

She, Jin 263

Sheikhani, Ali 551

Shiau, A. C. 217, 381

Short, Richard 619

Smith, Leon 665

Smith, Ryan L. 55

Soejoko, D. S. 381, 493

Solhjoei, Nosratollah 569

Srivastava, R. 217, 493

Stanwell, Peter 39

Stefanoyiannis, A. P. 7, 217

Suh, T. S. 381

Sun, Jidi 39

Sun, Yuqing 755

Suriyapee, S. 381

Sutherland, B. 503

Swati, D. 413

Sylvander, S. R. 119

\section{T}

Takau, Viliami 325

Tang, F. 217

Tariq, Anam 643

Temurtas, Feyzullah 229

Tesneli, Ahmet Y. 229

Thayalan, K. 217

Thwaites, D. I. 357

Toh, H. J. 381

Toossi, Mohammad Taghi Bahreyni 83

Tran, Thu 205, 479

Trapp, J. V. 289, 357, 369

Truant, D. 255

TsedenIsh, Bolortuya 493

Tye, Susannah 157

\section{$\mathbf{U}$}

Ullah Yasin, Ubaid 643

Ung, N. M. 493

Usman Akram, M. 643
V

Vafaeyan, H. 611

Vand, Safa Rafiei 551

Vetri Selvi, G. Uma 731

Vinijsorn, S. 381

Vinod, Shalini K. 561

Voon, E. O. 493

W

Wang, Bu-Hai 767

Wang, Xiao-Lei 767

Wang, Yilong 785

Wang, Yongjun 785

Webb, David 325

Whelan, Brendan 561

Williams, Ivan 151

Win, U. M. 493

Wong, Kelvin K. L. 375, 377, 743

Wright, Tracy 325

Wu, Jianhuang 627

Wu, Wan-Qing 709

$\mathrm{Wu}$, Zhanglin 109

$\mathbf{X}$

Xia, Lianghua 755

Xiang, Chunling 743

Xie, Lizhe 345

$\mathrm{Xu}$, Dachuan 695

$\mathrm{Xu}$, Jing 109

Y

Yan, Bin 109

Yang, Benqiang 345

Yang, Jian 345

Yang, Jun 263

Yang, Yang 109

Ye, Xuelang 695

Yin, Xindao 345

Younus Javed, M. 643

Yu, L. 119

Yu, Mengsun 263

Yu, Shaoxiao 695

Yu, Zhi-Ying 709

$\mathbf{Z}$

Zafar, H. 593

Zafar, J. 473, 593

Zafar, T. 473, 593

Zakavi, Seyed Rasoul 465

Zarafshan, Hadi 551

Zeng, Canjun 109, 743 
Zeng, Jinhao 695

Zhang, Bo 755

Zhang, Da 263

Zhang, Guodong 109

Zhang, Libo 345
Zhang, Runhua 785

Zhang, Zhenwei 695

Zhao, Xingquan 785

Zheng, Buhong 271 